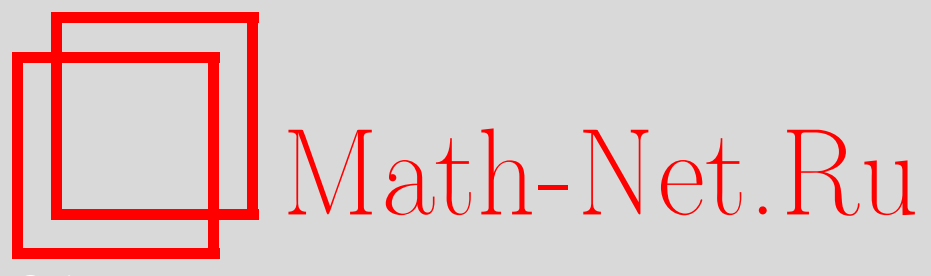

В. А. Зорич, Теорема о глобальном гомеоморфизме для конформно-гиперболических многообразий, УМH, 2007, том 62, выпуск 4, 159-160

DOI: https://doi.org/10.4213/rm7152

Использование Общероссийского математического портала Math-Net.Ru подразумевает, что вы прочитали и согласны с пользовательским соглашением http://www . mathnet.ru/rus/agreement

Параметры загрузки:

IP : 34.229 .45 .116

26 апреля 2023 г., 14:14:24

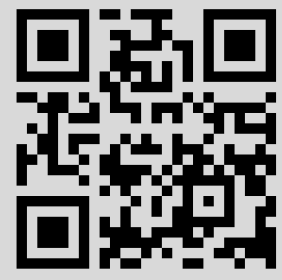




\title{
Теорема о глобальном гомеоморфизме для конформно-гиперболических многообразий
}

\author{
В. А. Зорич
}

1. Введение. Локально обратимое квазиконформное отображение $f: \mathbb{R}^{n} \rightarrow \mathbb{R}^{n}$ при $n>2$ обратимо глобально.

Это утверждение (часто называемое теоремой о глобальном гомеоморфизме для квазиконформных отображений) было сформулировано М. А. Лаврентьевым [1] для $n=3$ и позднее доказано в работе [2]. Затем оно было развито в нескольких направлениях (см. обзоры [3], [4]).

Переход от погружений евклидова пространства к общему случаю погружений римановых многообразий [5], [6] стимулировал появление следующей конформноинвариантной формы исходной теоремы, связанной с асимптотической геометрией и конформной классификацией римановых многообразий [7].

Для глобальной инбективности квазиконформного погружения $f: M^{n} \rightarrow N^{n} p u$ манова многообразия $M^{n}$ в односвязное риманово многообразие $N^{n}$ nри $n>2$ достаточно, чтобы многообразие $M^{n}$ имело конформно-параболический тип.

В [3], [4], однако, высказывалось предположение, что теорема о глобальном гомеоморфизме, по-видимому, имеет место и для определенных классов многообразий конформно-гиперболического типа.

Здесь мы хотим привести пример такого типа теоремы и высказать некоторые соображения, касающиеся общего случая.

\section{2. Формулировка основного результата.}

Теорема. Пусть $D^{k}$ - диск размерности $k \leqslant n-2$ в пространстве $\mathbb{R}^{n} ; U$ - некоторая окрестность множества $D^{k}$ в $\mathbb{R}^{n} ; f: U \rightarrow \mathbb{R}^{n}$ - непрерывное отображение, локально обратимое и квазиконформное вне $D^{k}$. Если $n>2$, то отображение $f$ обратимо в некоторой окрестности каждой точки множества $D^{k}$.

СледствиЕ. Если непрерывное отображение $f: \mathbb{R}^{n} \rightarrow \mathbb{R}^{n}$ локально обратимо и квазиконформно вне некоторого отрезка, диска $D^{k}$ конечного радиуса, луча или $k$-мерной полуплоскости с краем, то при $n>2 u k \leqslant n-2$ отображение $f$ обратимо глобально (т.е. $f$ - квазиконформная биекиия).

3. Схема доказательства. Из общих свойств квазирегулярных отображений (см., например, [8], [9]) следует, что отображение $f$ квазирегулярно в $U$ и имеет конечный индекс в каждой точке особого множества $D^{k}$.

Учитывая это, из топологических соображений сначала получим локальную обратимость отображения $f$ в точках края диска $D^{k}$. После этого, очевидно, можно будет перейти к меньшему множеству и, повторяя процедуру, свести все к уже известному случаю [4], [10], когда диск вырождается в точку.

Для наглядности разберем сначала ситуацию, когда $k=1$, а $n=3$ (конечный отрезок $I$ прямой в трехмерном пространстве).

Пусть $o$ - точка края (конец) нашего отрезка. Рассмотрим сферу $S^{2}(o, r)$ радиуса $r$ с центром $o$. Если радиус $r$ достаточно мал, то отображение $f$ не принимает на $S^{2}(o, r)$ значения $f(o)$ и ограничение $f$ на $S^{2}(o, r)$ локально инъективно всюду, кроме точки пересечения сферы $S^{2}(o, r)$ и отрезка $I$. Образ отображения $f \mid S^{2}(o, r)$ вне точки $f(o)$ можно гомотопировать в сферу с центром $f(o)$, сохраняя свойство локальной инъективности отображения $f$ (точнее, гомотопируемого отображения). Тогда получим непрерывное отображение сферы $S^{2}$ в себя, локально инъективное

Работа выполнена при поддержке грантов РФФИ 05.01.00981 и НШ-9429.2006.1. 
вне единственной точки, что возможно только, когда оно - гомеоморфизм. Но тогда ограничение исходного отображения $f$ на шар $B^{3}(o, r)$, сохраняя ориентацию, имеет индекс 1 , т. е. локально гомеоморфно.

Если $k=n-2$, то при $n \geqslant 3$ можно теперь действовать по индукции, учитывая, что пересечение малой гиперсферы пространства $\mathbb{R}^{n}$ с центром в точке края особого множества (диска $D^{k}$ ) и самого этого множества с топологической точки зрения есть $D^{k-1}$.

Если же $k<n-2$, то при $n \geqslant 3$ дело упрощается тем, что удаление множества коразмерности 3 и выше не меняет фундаментальную группу многообразия, в частности, не меняет его односвязности, если оно исходно было таковым. В нашем случае рассматривается сфера, и можно непосредственно воспользоваться теоремой о монодромии в ситуации, когда имеется непрерывное отображение сферы в себя, локально инъективное вне множества коразмерности 3 или выше.

Следствие теперь получается прямой ссылкой на исходную теорему о глобальном гомеоморфизме, приведенную в начале этой заметки.

4. Комментарий. В теореме мы полагали, что исходное отображение определено и непрерывно на особом множестве $D^{k}$, хотя есть основания считать, что эти предположения излишни. Например, в [9] показано, что если ограниченное квазирегулярное отображение определено вне некоторого множества достаточно большой коразмерности, то отображение автоматически продолжается на такое множество, оставаясь квазирегулярным.

В теореме указан модельный случай особого множества - диск $D^{k}$ размерности $k \leqslant n-2$. Ясно, однако, что топологический эквивалент $D^{k}$ тут тоже допустим.

Полезным дополнением к рассмотренной теореме была бы сформулированная ниже лемма. В сочетании с уже развитой при доказательстве исходной теоремы о глобальном гомеоморфизме техникой, она позволяет доказать аналогичную теорему, например, в случае отображения в себя односвязного многообразия размерности $n \geqslant 3$, имеющего абсолют размерности $k<n-1$.

Лемма. При непостоянном квазирегулярном отображении открытых римановых многообразий размерность абсолюта многообразия образа не превосходит размерности абсолюта многообразия прообраза.

\section{Список литературы}

[1] М. А. Лаврентьев, Докл. АН СССР, 20:4 (1938), 241-242. [2] В. А. Зорич, Матем. сб., 74:3 (1967), 417-433. [3] V. A. Zorich, Quasiconformal space mappings, Lecture Notes in Math., 1508, Springer, Berlin, 1992, 132-148. [4] В. А. Зорич, УМH, 57:3 (2002), 3-28. [5] M. Gromov, Riemann surfaces and related topics: Proceedings of the 1978 Stony Brook Conference (Stony Brook, 1978), Ann. of Math. Stud., 97, Princeton Univ. Press, Princeton, NJ, 1981, 183-213. [6] M. Gromov, Metric structures for Riemannian and non-Riemannian spaces, Progr. Math., 152, Birkhäuser, Boston, 1999. [7] В. А. Зорич, Функи. анализ и его прилож., 34:3 (2000), 37-48. [8] Ю. Г. Решетняк, Пространственные отображения с ограниченным искажением, Наука, Новосибирск, 1982. [9] T. Iwaniec, L. Migliaccio, L. Nania, C. Sbordone, Math. Scand., 75:2 (1994), 263-279. [10] В. А. Зорич, УМН, 56:4 (2001), 147-148.

В. А. Зорич (V. A. Zorich)

Московский государственный университет им. М. В. Ломоносова

E-mail: vzor@glasnet.ru
Представлено Д. В. Аносовым Принято редколлегией 08.05.2007 